\title{
A Performance Evaluation of SAR-GMTI Missions for Maritime Applications
}

\author{
Eduardo Makhoul, Student Member, IEEE, Antoni Broquetas, Member, IEEE, Josep Ruiz Rodon, Student \\ Member, IEEE, Yu Zhan, Francisco Ceba
}

\begin{abstract}
This paper proposes a new optimized multichannel synthetic aperture radar (SAR) configuration, based on receiving antennas with non-uniformly displaced phase centers, intended for ground moving target indication (GMTI) applications over maritime scenarios. This system is compared with current SAR missions, such as TerraSAR-X (TSX) or TanDEM-X (TDX). The GMTI capabilities of the different configurations are analyzed in a two-level performance approach. First, an intensive numerical simulation evaluation, based on Monte Carlo (MC) trials, is carried out in order to characterize the probabilities of detection under different system parameters as well as scenario conditions. Different GMTI techniques, displaced phase center antenna (DPCA), along-track interferometry (ATI) and extended displaced phase center antenna (EDPCA), are assessed. In a second step, synthetic simulated SAR data, obtained in a study case scenario, is used to demonstrate the potential improvement of the proposed multichannel configuration compared to current SAR missions, providing subclutter visibility for maritime surveillance of small and slowly moving boats.
\end{abstract}

Index Terms-Synthetic aperture radar (SAR), ground moving target indication (GMTI), displaced phase center antenna (DPCA), along-track interferometry (ATI), extended DPCA (EDPCA), constant false alarm rate (CFAR) detector, Monte Carlo (MC) simulations, SAR raw data simulation.

\section{INTRODUCTION}

$\mathbf{S}$ YNTHETIC aperture radar (SAR) systems for remote sensing applications have been gaining special interest during the last decade, as demonstrated by the increased number of missions: TerraSAR-X (TSX), TanDEM-X (TDX), COSMO-SkyMed, RADARSAT-2 (RS2), Sentinel-1 and PAZ [1]-[4]. Some of them include an experimental mode that adds ground moving target indication (GMTI) capabilities from spaceborne platforms. This kind of missions will provide a powerful tool to globally monitor traffic, illegal ship movements, migration movement and piracy attacks, covering the requirements on the so called Situation Awareness, [5]-[8].

Single channel approaches, based on Doppler filtering, fail to detect slow moving targets, [9]. Moreover, an intrinsic

Manuscript received Month Day, Year; revised Month Day, Year.

This work has been supported by FPU Research Fellowship Program, Ministerio de Educación, contract AP2009-4590; by FPI-AGAUR Research Fellowship Program, Generalitat de Catalunya, contract 2010FI EM051757; by the Spanish Ministry of Science and Innovation (MICINN) under projects TEC2011-28201-C02-01 and CONSOLIDER CSD2008-00068; and by European Commission under FP7-SPACE Project SIMTISYS Ref. 263268.

E. Makhoul, A. Broquetas, J.Rodon, Y. Zhan and F.Ceba are with the Department of Signal Theory and Communications, Universitat Politècnica de Catalunya, Barcelona (Spain). Phone: +34 934017359, e-mail: \{edumak,broquetas,josep.ruiz,yu.zhan,francisco.ceba\} @ tsc.upc.edu ambiguity between radial velocities and azimuth location in single SAR images exists, [9]. This ambiguity problem can be solved by means of multiple receiving channels, which provide improved detection capabilities thanks to the increased spatial diversity. Current SAR missions, as TSX, TDX and RS2, equipped with two receiving antennas, have limited capabilities to detect weak and slow moving targets, i.e. small boats in the sea. For this dual receive antenna (DRA) mode configurations, conventional SAR-GMTI processing techniques operating on the SAR images can be applied, based on either phase change detection, along-track interferometry (ATI) [10][11]; or clutter cancellation in the case of displaced phase center antenna (DPCA) [11]-[13].

In order to obtain adequate performance, by means of spacetime adaptive processing (STAP), more than two receiving antennas are required [9]. One way to obtain additional spatial diversity, without increasing the system complexity, is based on the use of suitable antenna switching and toggling modes [14]. These kinds of approaches imply an antenna effective area reduction, resulting in a degradation of the signal-to-noise ratio (SNR) and increased pulse repetition frequency (PRF) requirements to reduce the azimuth ambiguities, [14].

Alternative multichannel approaches are based on the deployment of coherently operating SAR constellations, which provide better performances (angular and Doppler resolutions) thanks to extended apertures [15], [16]. However, this kind of configurations suffer from a high number of grating lobes, creating numerous blind velocities. Moreover, the technological complexity and cost of maintaining a constellation of satellites is rather high. From these considerations, this manuscript proposes an optimized SAR mission that maximizes the GMTI performance, keeping low system complexity in terms of receiving channels and hence data volume to be downloaded. The novel architecture is based on a single monostatic multichannel satellite with non-uniformly spaced receiving antennas, as briefly discussed in [7] and [17]. The objective is to provide subclutter visibility for maritime surveillance of small slowly moving boats. This optimized multichannel configuration, in combination with the new optimum GMTI processing techniques as imaging STAP (ISTAP) and extended DPCA (EDPCA), is expected to provide a substantial improvement in SAR-GMTI performance, [18]-[19].

This paper presents an exhaustive GMTI performance evaluation of the proposed multichannel configuration compared to current state-of-the-art SAR missions over maritime scenarios. The GMTI capabilities are analyzed and characterized in terms 
of probability of detection based mainly on intensive numerical simulations at image level. In Section II, the multichannel data model at SAR image level is described, specifying the different assumed hypotheses. The different GMTI techniques evaluated throughout the paper (DPCA, ATI and EDPCA) are briefly presented in Section III. Section IV analyzes comparatively the expected theoretical SAR-GMTI performance of the different X-band configurations. Section V shows the results of the intensive numerical simulation based on Monte Carlo trials, which are complemented with processed synthetic raw data, for a representative sea clutter scenario. Through the manuscript a Gaussian-like model for the sea clutter is assumed. For analysis completeness, additional processed synthetic raw data results are included when considering a nonGaussian sea clutter based on the K-distribution, [20]. Both Gaussian and K-distributions have been observed analyzing real data from TSX dual- and quad-polarized modes, see [21]. A preliminary study on the impact of SAR imaging high-speed boats (with high induced vertical and horizontal accelerations) has been also carried out, considering a complete SARGMTI processing chain, which integrates a matched filter bank (MFB) based on an adaptive range-Doppler (RD) processor. The different theoretical and simulation performance evaluations prove the potential capabilities of the proposed SARGMTI mission compared to current state-of-the-art missions.

\section{DATA MODEL}

Consider a general multichannel SAR configuration, consisting of $M$ parallel receiving (RX) antennas collocated in the along-track direction $x$, which are displaced $\left\{d_{x_{m}}\right\}_{m=1}^{M}$ from the transmitting (TX) reference phase center. A flat Earth geometry is assumed in the modeling, such that the platform, orbiting at a constant height $H_{\text {orb }}$, moves with an effective velocity $v_{e}$, [22].

The detection of the moving target, whose multichannel signal is denoted by $\mathbf{s}\left(\boldsymbol{\vartheta}_{t}\right)$, can be understood as a hypothesis testing problem

$$
\begin{aligned}
& H_{1}: \mathbf{x}=\mathbf{s}\left(\boldsymbol{\vartheta}_{t}\right)+\mathbf{c}\left(\boldsymbol{\vartheta}_{c}\right)+\mathbf{n} \\
& H_{0}: \mathbf{x}=\mathbf{c}\left(\boldsymbol{\vartheta}_{c}\right)+\mathbf{n},
\end{aligned}
$$

driven by the presence of interference $\mathbf{q}$, which consists of background clutter $\mathbf{c}\left(\boldsymbol{\vartheta}_{c}\right)$ and thermal noise n. $\boldsymbol{\vartheta}_{t}=$ $\left\{v_{x}, v_{z}, R_{0}, \sigma_{t}^{2}\right\}$ and $\boldsymbol{\vartheta}_{c}=\left\{v_{x_{c}}, v_{z_{c}}, R_{0}, \sigma_{c}^{2}\right\}$ are parameter vectors of the moving target and clutter, respectively; where the first two elements of the vectors correspond to the alongand across-track velocities, with $R_{0}$ the slant range of closest approach and the related power levels indicated by the sigma terms.

\section{A. Signal}

The target, modeled as a collection of single-like point scatterers, moves on the ground plane $(y=0)$ with a uniformly accelerated movement in both along- $(x)$ and across-track $(z)$ directions, as generally assumed in the literature [11], [23]:

$$
\begin{aligned}
& x(t)=x_{0}+v_{x} t+\frac{a_{x}}{2} t^{2} \\
& z(t)=z_{0}+v_{z} t+\frac{a_{z}}{2} t^{2},
\end{aligned}
$$

where $t$ stands for slow time (or azimuth time). At $t=0$ the target is located at $x_{0}$ and $z_{0}$, which corresponds to the alongand across-track coordinates, respectively. Target velocities and accelerations are, respectively, represented by $v_{x}, v_{z}$, and $a_{x}, a_{z}$.

The multichannel signal of the moving target, at resolution cell level, can be formulated using vectorial notation as:

$$
\mathbf{s}\left(\boldsymbol{\vartheta}_{t}\right)=\alpha_{t} \boldsymbol{\Delta}_{A T I}\left(\boldsymbol{\vartheta}_{t}\right),
$$

where the different SAR images have been properly coregistrated and balanced, [24]. It is well-known that the target radar cross section (RCS) varies with the aspect angle [25]. For the deterministic target model the different scattering centers are assumed to have a constant radar response during the integration time, such that for calibrated images, $\alpha_{t}$ is directly related to the square root of the RCS. The term $\boldsymbol{\Delta}_{A T I}\left(\boldsymbol{\vartheta}_{t}\right)$ collects the along-track phase change for the different channels at the displaced image position of the moving target. As it is extendedly considered in literature [23]-[25], $x_{0}=0$ is assumed for simplicity in the mathematical notation without losing generality. Then, the interferometric phase component of the $i$-th channel can be expressed as [23]

$$
\angle\left[\boldsymbol{\Delta}_{A T I}\left(\boldsymbol{\vartheta}_{t}\right)\right]_{i}=\phi_{i}\left(\boldsymbol{\vartheta}_{t}\right)=\frac{\pi d_{x_{i}} v_{r}}{\lambda} \cdot\left[\frac{1}{v_{e}}-\frac{v_{x}-v_{e}}{v_{r e l}^{2}\left(\boldsymbol{\vartheta}_{t}\right)}\right],
$$

where $\lambda$ is the carrier wavelength; $v_{r}$ refers to the radial velocity, i.e. line-of-sight projection of the ground range velocity; and the relative velocity term $v_{r e l}$ is expressed as:

$$
v_{\text {rel }}\left(\boldsymbol{\vartheta}_{t}\right)=\sqrt{\left(v_{x}-v_{e}\right)^{2}+v_{z}^{2}\left(1-\frac{z_{0}^{2}}{R_{0}^{2}}\right)+z_{0} a_{z}} .
$$

A more realistic modeling, which accounts for the target's random variability on the radar response, is a zero-mean complex Gaussian scattering center, [26], i.e. $\alpha_{t} \in \mathbb{C} \sim$ $\mathcal{N}\left(0, \sigma_{t}^{2}=R C S\right)$. Then, the multichannel target signal $\mathbf{s}\left(\boldsymbol{\vartheta}_{t}\right)$ is characterized by its covariance matrix $\mathbf{R}_{t}$, where decorrelation effects induced either by the target motion itself or the internal clutter motion (ICM), [27], can be included through the target correlation coefficient between each pair $(i, j)$ of channels $\rho_{t_{i, j}}$, similar to the sea clutter in (6).

\section{B. Interference}

GMTI processing intends to detect moving targets masked by the presence of interference, consisting of the background clutter $\mathbf{c}\left(\boldsymbol{\vartheta}_{c}\right)$ and the unavoidable thermal noise $\mathbf{n}$ of the receiver. The latter is generally modeled as a zero-mean complex Gaussian process, $\mathbf{n} \in \mathbb{C} \sim \mathcal{N}\left(\mathbf{0}, \mathbf{R}_{n}\right)$. The noise has neither a temporal nor a spatial structure, $\mathbf{R}_{n}=\sigma_{n}^{2} \mathbf{I}$, where I stands for a diagonal unitary matrix. For calibrated SAR images the noise mean power is related to the noiseequivalent sigma zero (NESZ) and the ground resolution cell area.

The clutter has specific temporal and spatial correlations, which account for both temporal and spatial variability of its radar returns. Since the scope of the paper is to demonstrate the improved capabilities of the proposed configuration against current state-of-the-art SAR-GMTI missions, the clutter is 
assumed to have a stationary zero-mean complex Gaussian distribution $\mathbf{c}\left(\boldsymbol{\vartheta}_{c}\right) \in \mathbb{C} \sim \mathcal{N}\left(\mathbf{0}, \mathbf{R}_{c}\right)$, as it is extendedly considered in the literature [16], [18], [19]. This hypothesis breaks down when considering realistic scenarios that may contain highly heterogeneous terrains, such as urban or industrial areas [13]. In the maritime case, it has been assumed that a Kdistribution (compound model) fits well the sea clutter returns [20]. In [21], a preliminary statistical analysis of the sea clutter using two TSX data takes (dual- and quad-polarized) has been conducted, showing a good fitting of the K-distribution to the magnitude. For incidence angles between 29.7 and 32.3 degrees, shape parameters $v$ between 3-10 are observed (small values indicate spiky/highly heterogeneous returns). To complement the mission performance study, a K-distributed sea clutter has been also simulated for the synthetic raw data approach (section V-B) in order to understand the impact of non-Gaussian statistics.

A way to provide a more realistic maritime scenario is twofold: account for specific clutter decorrelation between the different channels induced by the ICM and the inclusion of an ATI phase, which can be related to a mean surface velocity on the sea structure. In this sense, the covariance matrix of the clutter $\mathbf{R}_{c}$ collects those parameters as:

$$
\begin{aligned}
& \mathbf{R}_{c}=E\left\{\mathbf{c}\left(\boldsymbol{\vartheta}_{c}\right) \mathbf{c}\left(\boldsymbol{\vartheta}_{c}\right)^{H}\right\}= \\
& {\left[\begin{array}{ccc}
\sigma_{c_{1}}^{2} & \ldots \rho_{c_{1, M}} \sqrt{\sigma_{c_{1}}^{2} \sigma_{c_{M}}^{2}} e^{\jmath \Delta \phi_{c_{1, M}}} \\
\vdots & \ddots & \vdots \\
\rho_{c_{1, M}} \sqrt{\sigma_{c_{1}}^{2} \sigma_{c_{M}}^{2}} e^{-\jmath \Delta \phi_{c_{1, M}}} \ldots & \sigma_{c_{M}}^{2}
\end{array}\right],}
\end{aligned}
$$

where $\sigma_{c_{i}}^{2}$ refers to the clutter power for the $i$-th channel, which is related to the radar backscattering coefficient $\sigma^{0}$ and the ground resolution cell area. For maritime scenarios, this coefficient can be obtained using the Naval Research Laboratory (NRL) model [28], given specific system parameters (frequency, polarization, incidence angle) and scenario conditions (sea state or wind velocity). The phase term $\Delta \phi_{c_{i, j}}$ represents the ATI phase between the $i$-th and $j$-th channels due to the sea mean Doppler velocity, [29].

In (6), the correlation coefficient $\rho_{c_{i, j}}$ between channels $i$ and $j$ takes into account the temporal decorrelation induced by the ICM and is related to the (baseline) time-delay $\tau_{i, j}$ between those two channels (equivalent two-way phase centers). From [27] and under the assumption of Gaussian distributed clutter, $\rho_{c_{i, j}}$ has a Gaussian-like shape:

$$
\rho_{c_{i, j}}=\exp \left\{-\left(\frac{\tau_{i, j}}{\tau_{c}}\right)^{2}\right\}=\exp \left\{-\left(\frac{\frac{\left(d_{x_{i}}-d_{x_{j}}\right) \cdot v_{e}}{2 \cdot v_{r e l}^{2}\left(\boldsymbol{\vartheta}_{c}\right)}}{\tau_{c}}\right)^{2}\right\},
$$

where $\tau_{c}$ is the clutter correlation (coherence) time, which for an X-band system can vary between 10-60 ms, depending on the sea conditions and system resolution, [29].

As the radar measures range and velocity (Doppler) ambiguously, the moving target should compete also with the ambiguous clutter patches. Therefore, for the main non-ambiguous clutter patch, a grid of $N$ surrounding ambiguous clutter responses should be also considered when modeling the clutter
[14], [18], [25]. Assuming a homogeneous (stationary) complex Gaussian sea clutter surface and uncorrelated ambiguities, the interference covariance matrix can be expressed as:

$$
\mathbf{R}_{q}=\mathbf{R}_{c_{0}}\left(\boldsymbol{\vartheta}_{c}\right)+\sum_{k, l}^{N} \mathbf{R}_{c_{k, l}}\left(\boldsymbol{\vartheta}_{c}\right)+\mathbf{R}_{n}, \quad(k, l) \in \mathbb{Z}^{2} \backslash\{0,0\},
$$

where $\mathbf{R}_{c_{0}}\left(\boldsymbol{\vartheta}_{c}\right)$ is the covariance matrix for the main ${ }^{1}$ clutter patch, as expressed in (6); analogously, $\mathbf{R}_{c_{k, l}}\left(\boldsymbol{\vartheta}_{c}\right)$ is the covariance matrix for the $k, l$-th ambiguous patch. In (8), $(k, l) \in \mathbb{Z}^{2} \backslash\{0,0\}$ denotes the set of those whole-numbered index pairs of admissible range-azimuth ambiguities except for $k=l=0$, which refers to the non-ambiguous clutter patch of interest. For simplicity the main clutter and the related ambiguous patches are assumed to have equal backscattering coefficient $\sigma^{0}$ and correlation properties. In this case, the corresponding power of the $k, l$-th ambiguous patch for the $i$-th channel is defined by

$$
\sigma_{c_{i}^{k, l}}^{2}=\sigma_{c_{i}}^{2} \cdot \operatorname{CRAASR}_{k, l},
$$

where $\mathrm{CRAASR}_{k, l}$ refers to the combined-range-azimuthambiguity-to-signal ratio for the $k, l$-th ambiguity, as defined in section IV-B, such that a SAR related figure of merit is incorporated into the GMTI performance by means of the covariance matrix modeling.

The off-diagonal phase terms of $\mathbf{R}_{c_{k, l}}\left(\boldsymbol{\vartheta}_{c}\right)$ are modeled as

$$
\Delta \beta_{i, j}^{k, l}=\Delta \phi_{c_{i, j}}-2 \pi \cdot l \cdot \operatorname{PRF} \cdot \frac{\left(d_{x_{i}}-d_{x_{j}}\right) \cdot v_{e}}{2 \cdot v_{r e l}^{2}\left(\boldsymbol{\vartheta}_{c}\right)},
$$

accounting for the interferometric phase $\Delta \phi_{c_{i, j}}$ induced by the clutter's mean Doppler velocity and the residual phase term due to wrong channel coregistration on the ambiguities as pointed out in [19]. This spatial alignment (coregistration) is accomplished by time-shifting the signals of the different channels via interpolation, efficiently performed applying a phase ramp in the Doppler domain. Due to partial backfolding of the different Doppler ambiguities (modeled by the $l$-th order azimuth ambiguity), this phase ramp produces a residual constant phase error on these ambiguities, modeled by the second phase term in (10). This residual error vanishes when the DPCA condition holds, i.e. the satellite's displacement between pulses is a multiple of the baseline separation between the involved channels.

\section{GMTI PROCESSING TECHNIQUES}

\section{A. Displaced Phase Center Antenna (DPCA)}

DPCA is a motion compensation technique, such that the equivalent two-way phase centers of the two antennas $(i-$ th and $j$-th) occupy the same spatial location but at different instants of time. Therefore, a simple complex subtraction between the two coregistrated channels allow canceling the clutter while the moving targets show up in the final DPCA image, [11][13].

Ideally, DPCA could completely remove the clutter down to noise floor, but actually, the clutter suppression is limited by

\footnotetext{
${ }^{1}$ From now on the subindex 0 refers always to the main clutter patch.
} 
possible ICM, coregistration errors and channel imbalances. Based on a constant false alarm rate (CFAR) detector, the DPCA magnitude is compared against a threshold $\xi$, which can be determined from the desired probability of false alarm $P_{f a}$ and the related residual interference probability density function (PDF), [12].

\section{B. Along-track interferometry (ATI)}

The high sensitivity of the interferometric phase to slight variations in the scene allows exploiting the ATI to perform detection of slowly moving targets [10]-[13]. ATI, as DPCA, is a dual-channel antenna technique, where the formation of the interferogram allows both detection of the moving target and estimation of its radial velocity. As opposed to DPCA, in the ATI processing there is no clutter cancellation. Therefore, the presence of both clutter and noise produces a noise phase that can impair the detection and estimation of the targets radial velocity. The ATI signal is formed as the complex conjugate multiplication of the two coregistrated channels

$$
\mathrm{ATI}_{\mathrm{i}, \mathrm{j}}=\frac{1}{n} \sum_{p}^{n}[\mathbf{x}]_{i, p}[\mathbf{x}]_{j, p}^{*},
$$

where $n$ corresponds to the number of looks (or resolution cells).

To evaluate the ATI performance, a CFAR detector exploiting the joint PDF of ATI magnitude $\eta$ and phase $\psi$ has been selected since it can provide improved performance compared to the two-step detectors (phase and magnitude separately, [10]), as pointed out in [30].

\section{Extended Displaced Phase Center Antenna (EDPCA) pro- cessing}

In [18], Cerutti et al. have proposed an optimized SARGMTI processing technique, ISTAP, which exploits a suboptimal post-Doppler STAP in combination with SAR processing, following a similar approach as in [9], [31]. Cerutti et al. have also proposed EDPCA [19], which is an adaptive DPCA technique for architectures with more than two receive channels.

Clutter homogeneity plays an important role in the estimation of the interference covariance matrix performed by ISTAP and EDPCA, and so directly affects the GMTI performance. In this sense, the EDPCA processing is preferred over maritime scenarios since it operates at image level, allowing to select and process homogeneous patches. Moreover, the performance characterization of the ISTAP via MC simulations would require its evaluation in the range-Doppler domain leading to a much more computationally costly approach compared to the efficient one presented in section V-A, which operates at image level. Both ISTAP and EDPCA provide the same GMTI performance when the DPCA condition is fulfilled; otherwise, ISTAP gives improved results as pointed out in [18].

Assuming that the $M$ SAR images have been properly coregistrated and SAR focused with the target parameters $\vartheta_{t}$ (except radial velocity shift compensation), the weighting vector that provides the optimal combination of the different channels in terms of SCNR maximization at the EDPCA processor's output, $y=\mathbf{w}^{H}\left(\boldsymbol{\vartheta}_{t}\right) \mathbf{x}$, is [32]:

$$
\mathbf{w}\left(\boldsymbol{\vartheta}_{t}\right)=\beta \mathbf{R}_{c}^{-1} \mathbf{d}\left(\boldsymbol{\vartheta}_{t}\right),
$$

where $\mathbf{d}\left(\boldsymbol{\vartheta}_{t}\right)$ refers to the spatial beamformer adapted to the target parameters, such that the phase differences between the channels are compensated

$$
\mathbf{d}\left(\boldsymbol{\vartheta}_{t}\right)=\boldsymbol{\Delta}_{A T I}\left(\boldsymbol{\vartheta}_{t}\right) .
$$

In order to ensure a CFAR test statistics at the output of the EDPCA, the scalar term $\beta$ in (12) is properly chosen as:

$$
\beta=\frac{1}{\sqrt{\mathbf{d}^{H}\left(\boldsymbol{\vartheta}_{t}\right) \mathbf{R}_{c}^{-1} \mathbf{d}\left(\boldsymbol{\vartheta}_{t}\right)}},
$$

ensuring a unitary power residual interference. Similar to DPCA, the magnitude at the output of EDPCA should be compared against a threshold for a given $P_{f a}$.

\section{MISSION ANALYSIS}

\section{A. Multichannel configurations}

The state-of-the-art SAR missions, as TSX, TDX or RS2, equipped with two receiving antennas, have limited GMTI capabilities to detect slowly moving targets, due to both, the reduced number of channels and to the short baselines (2.4 $\mathrm{m}$ one way for TSX), which determine the minimum detectable velocity. The deployment of coherently operating SAR constellations, as TDX, provides improved performance in terms of Doppler (velocity) and angular resolution, but at expense of a reduced range of unambiguous velocities (around $1.4 \mathrm{~m} / \mathrm{s}$ ), caused by the longer baselines (around 200 $\mathrm{m})$. In the maritime scenarios, where the clutter correlation could reach tens of milliseconds [29], depending on the sea conditions, the channel coherence (for the longer baseline) could drop off dramatically, and so would the capability of clutter rejection. Therefore, a trade-off solution is to consider a monostatic multichannel configuration with non-uniformly spaced receivers, taking advantage of baseline diversity to ensure high sensitivity to slowly moving targets (with the largest baseline), and simultaneously alleviating the Doppler ambiguities (with the shorter ones); in a way that channel coherence can be kept high enough to ensure proper detection capabilities.

From these considerations, a three-channel configuration is proposed (see Fig. 1(a)), where the two external antennas are deployed using a telescopic boom, as suggested in [7], [17], and as an unfolding system, respectively. The number of channels has been selected to limit system complexity and at the same time to fulfill the required degrees of freedom driven by the eigenvalue distribution of the interference covariance matrix, as discussed in section IV-B.

The length of the boom has been selected to deliver a probability of detection $\left(P_{d}\right)$ close to one for a $1 \mathrm{~m}^{2} \mathrm{RCS}$ target, whose ground range velocity is greater than $5.5 \mathrm{~m} / \mathrm{s}$ when processing the three-channel data cube with EDPCA. The boom length is a multiple of the antenna length assuming a telescopic structure will be used for deployment. The system operates in X-band, equipped with a configurable $4.8 \mathrm{~m}$ 


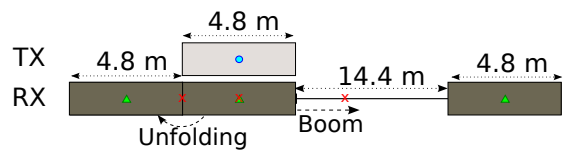

(a)

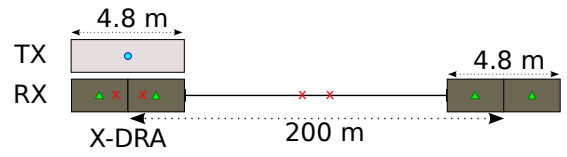

(b)

Fig. 1. Schematic representation of the different configurations to be analysed: (a) X-band Boom system and (b) Tandem system (including XDRA satellite); transmitting (TX) phase center denoted by the solid circle, receiving (RX) by the triangle symbol and effective two-way (2-W) by the cross symbol.

TABLE I

RADAR SYSTEM PARAMETERS

\begin{tabular}{lcc}
\hline Parameter & Value & Units \\
\hline Effective velocity $\left(\gamma_{0}=33.17 \mathrm{deg}\right)$ & 7311.6 & $\mathrm{~m} / \mathrm{s}$ \\
TX/RX antenna height & 0.7 & $\mathrm{~m}$ \\
Carrier frequency & 9.65 & $\mathrm{GHz}$ \\
Peak transmitted power & 2.2 & $\mathrm{KW}$ \\
Polarization & $\mathrm{HH}$ & \\
Noise factor & 4.3 & $\mathrm{~dB}$ \\
Transmitter $(\mathrm{TX}) /$ Receiver $(\mathrm{RX})$ losses & $2 / 2$ & $\mathrm{~dB}$ \\
\hline
\end{tabular}

phased-array antenna, with $32 \times 12$ transmit receive modules (TRMs). The main radar mission parameters, similar to TSX, are summarized in Table I. From now on, the proposed configuration will be referred as Boom system, and its SARGMTI performance will be compared against two different systems that emulate current state-of-the-art SAR missions: a $4.8 \mathrm{~m}$ length single satellite operating in dual receive antenna (DRA) mode (2.4 m per receiver), named as X-DRA; and a Tandem configuration, where two X-DRA fly in formation (only one TX) with a baseline of $200 \mathrm{~m}$ as schematically represented in Fig. 1(b). These systems are also characterized by the same radar parameters as in Table I.

\section{B. SAR-GMTI expected performance}

An orbital height of $514 \mathrm{Km}$ has been selected to ensure an incidence range coverage from 14 to 60 degrees (swath ground extension of $640 \mathrm{Km}$ ), intended to be covered by 27 subswaths, whose extension is in the order of $30 \mathrm{Km}$. A proper PRF selection has been carried out taking the highest possible ones (according to the timing/diamond diagram) while keeping a trade-off between the azimuth and range ambiguities level. For the proposed system, and assuming uniform tapering on the TRMs, the NESZ is kept below $-20 \mathrm{~dB}$ for the typical SAR incidence angles, 20-40 degrees. Instead, for low-grazing angles the noise contribution increases up to $-17 \mathrm{~dB}$. The optimization of the system's NESZ is conservative due to power budget restrictions, keeping a nominal peak transmitted power of $2.2 \mathrm{KW}$. It must be noted that the system operates with a pulse bandwidth of $150 \mathrm{MHz}$ for beams 1-10 (1438 degrees incidence angle) and $75 \mathrm{MHz}$ for the rest of the subswaths.

As modeled in section II-B, and noted in [14] and [25], the contribution of the ambiguous clutter returns should be taken into consideration as they could play an important role in the SAR-GMTI performance: the residual coregistration error on the ambiguities, as denoted by the second term in (10), when the DPCA condition does not hold, produces additional eigenvalues different from the noise floor, and therefore more RX channels are required to cancel out both unambiguous and ambiguous clutter patches, [19]. Moreover, temporal decorrelation of the sea clutter returns, present also in the ambiguous patches, could produce additional eigenvalues different from noise floor. These impacts depend in turn on the level of ambiguous returns with respect to (w.r.t.) the main clutter. The new combined-range-azimuth-ambiguity-tosignal ratio (CRAASR) metric is a helpful indicator: provides a single SAR performance metric that combines the definition of both the AASR as well as the RASR [33] for the grid of $N$ ambiguities:

$$
\begin{aligned}
\operatorname{CRAASR}= & \sum_{k, l}^{N} \operatorname{CRAASR}_{k, l}, \quad(k, l) \in \mathbb{Z}^{2} \backslash\{0,0\} \\
= & \sum_{k, l}^{N} \frac{R_{0}^{3} \cdot \sin \left(\gamma_{0}\right) \cdot\left|D_{2-w}\left(\theta_{k, l}, \phi_{k, l}\right)\right|^{2}}{R_{k, l}^{3} \cdot \sin \left(\gamma_{k, l}\right) \cdot\left|D_{2-w}\left(\theta_{0}, \phi_{0}\right)\right|^{2}} \\
& \cdot \frac{\int_{-B_{p} / 2}^{B_{p} / 2}\left|P_{2-w}\left(f_{d}+l \cdot \mathrm{PRF}\right)\right|^{2} d f}{\int_{-B_{p} / 2}^{B_{p} / 2}\left|P_{2-w}\left(f_{d}\right)\right|^{2} d f}
\end{aligned}
$$

where $R_{0}$ and $R_{k, l}$ correspond to the slant range for the main and the $k, l$-th ambiguous clutter patches, respectively; $\gamma_{k, l}$ refers to the incidence angle for the $k, l$-th ambiguous clutter patch. The impact of the two-way antenna patterns is accounted for in the $D_{2-w}\left(\theta_{k, l}, \phi_{k, l}\right)$ term, where $\theta_{k, l}$ and $\phi_{k, l}$ are the spherical angles in the antenna coordinate system for the specific ambiguous clutter patch. The term $P_{2-w}\left(f_{d}\right)$ models the Doppler spectrum, including the antenna pattern and the azimuth window used in SAR focusing for a specific $B_{p}$ processing bandwidth.

Considering the first $N=440$ combined ambiguities, a Kaiser window (factor of 2.5) with processing bandwidths of $2.8 \mathrm{KHz}$ in azimuth and the corresponding pulse bandwidth (subswath dependent) in range, the CRAASR for the Boom configuration is kept below $-20 \mathrm{~dB}$ for the range of interest (20-40 degrees incidence angle). However, the ambiguities' impact increases (up to $-5 \mathrm{~dB}$ ) for beams with high incidence angle (above 50 degrees), mainly due to range ambiguities. Nevertheless, assuming the ambiguous clutter returns have the same $\sigma^{0}$ as the main patch (close to the noise level for those incidence angles), the impact of the ambiguities gets masked by the noise floor.

Fig. 2(a) and Fig. 2(b) show the eigenvalue distribution of $\mathbf{R}_{q}$ as a function of the incidence angle for the Boom and Tandem configurations, respectively. This kind of chart is a good indicator of the interference distribution as a function of the incidence angle and so of the predominant mechanism, against which moving targets should compete. A flat distribution of the eigenvalues (similar values) indicates that a predominant noise-like effect is expected, either due to the high contribution of noise or due to the possibly combined impact of high clutter decorrelation and ambiguities contribution. 


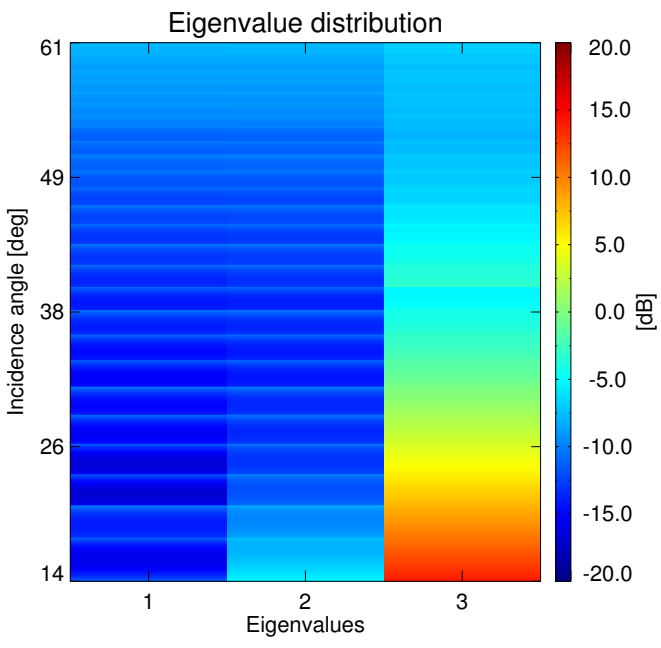

(a)

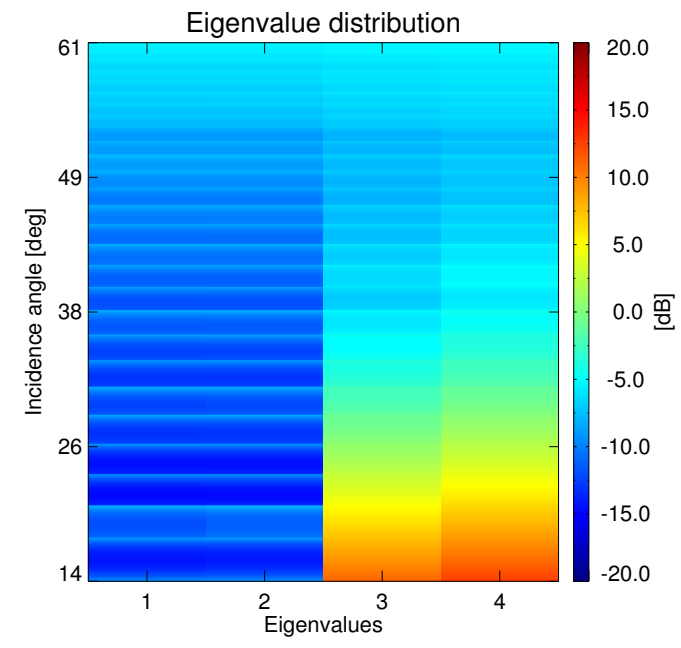

(b)

Fig. 2. Eigenvalue distribution for a $10 \mathrm{~ms}$ clutter coherence time with sea state 4 versus incidence angle: (a) Boom and (b) Tandem.

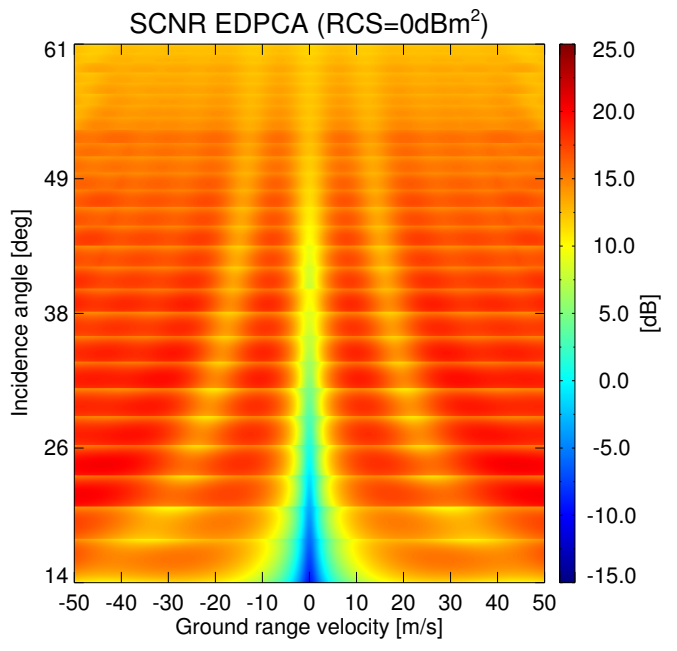

(a)

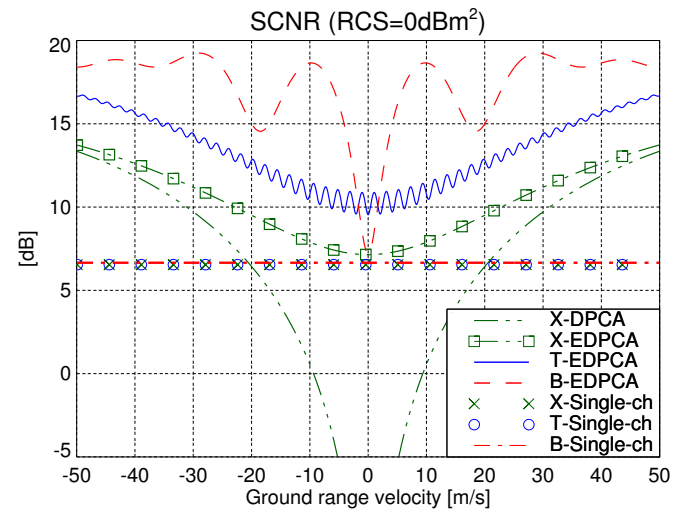

(b)

Fig. 3. Boom system SAR-GMTI theoretical performance for a deterministic target (RCS of $0 \mathrm{dBm}^{2}$ ) and a sea state 4 (Gaussian) clutter with $10 \mathrm{~ms}$ coherence time: (a) EDPCA-SCNR versus incidence angle and ground range velocity; (b) SCNR system-technique comparison at beam center of subswath 8 with 33.17 degrees of incidence angle (in legend X refers to X-DRA system, T and B to Tandem and Boom configurations, respectively).

For Boom configuration, Fig. 2(a), and incidence angles between 14 and 40 degrees, there is a predominant eigenvalue, such that two receive channels are sufficient to cancel out the clutter and its ambiguities. For higher incidence angles, noise contribution masks clutter response (the three eigenvalues are similar). Comparatively, for Tandem, Fig. 2(b), two predominant eigenvalues are present due to the major impact of clutter decorrelation, [32], induced by the longer baseline configuration: SAR-GMTI techniques at image level require channel coregistration, by means of a time-shift related to the baseline time-delay $\tau_{i, j}$, such that they observe the scene from the same position but at different instants of time. During this time frame sea has evolved, and so clutter returns have decorrelated from a statistical standpoint. For the longest baseline separation and $10 \mathrm{~ms}$ coherence time, sea clutter coherence drops off to values around 0.15 according to (7). This is directly translated into a GMTI performance degradation, as pointed out by the theoretical and simulation results.

In Fig. 3(a) EDPCA SCNR for the Boom system is plotted as a function of the incidence angle (beam) and ground range velocity ${ }^{2}$. For incidence angles below 38 degrees, a notch around the zero-radial velocity can be recognized, which for higher incidence angles is not present as the thermal noise starts to be the dominant interference contributor, in accordance to the eigenvalue distribution in Fig. 2(a).

Fig. 3(b) shows the SCNR cuts as a function of ground range velocity and at beam center of subswath 8 for different combination of system-techniques. From now on the comparative performance analysis is considered for a given set of system-technique combinations: classical (2-channel) GMTI techniques, such as DPCA and ATI, are presented only for X-

\footnotetext{
${ }^{2}$ The edge-like effects along the incidence angle are related to beam transitions (superposition between beams is not represented).
} 


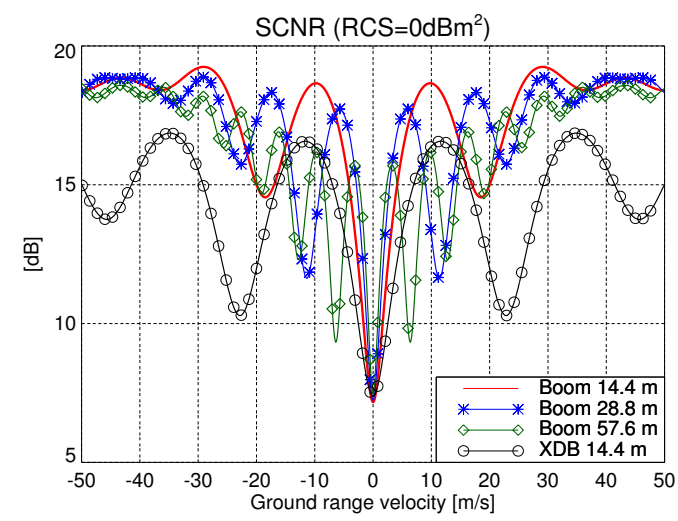

Fig. 4. SCNR cut as a function of ground range velocity (at center of subswath 8) for EDPCA technique considering different variants of the Boom configuration, assuming a deterministic target (RCS of $0 \mathrm{dBm}^{2}$ ) and a sea state 4 (Gaussian) clutter with $10 \mathrm{~ms}$ coherence time; XDB corresponds to a Boom configuration where the two first channels are X-DRA like $(2.4 \mathrm{~m}$ separation with no unfolding) and the third one (2.4 $\mathrm{m}$ antenna length) is located on a $14.4 \mathrm{~m}$ mast.

DRA system, showing the available capabilities for the current state-of-the-art SAR-GMTI missions with two RX channels; the optimum ( $>$ two RXs) EDPCA technique is considered for Boom and Tandem configurations to demonstrate the potentiality of oncoming SAR-GMTI architectures.

Processing with EDPCA the data cube of the proposed Boom system (dash red line) provides improved SCNR when compared to DPCA and EDPCA techniques applied to the $\mathrm{X}$-DRA system, dash-dot-dot green lines with and without square markers, respectively. The proposed Boom system proves improved detection capabilities, being more robust to clutter induced decorrelation effects, compared to Tandem system. The corresponding EDPCA SCNR (solid blue line) has a similar trend as X-DRA but with a mean SCNR 3 $\mathrm{dB}$ higher thanks to the two additional spatial degrees of freedom (DoF). At this point it must be also noted that for $v_{z}=0 \mathrm{~m} / \mathrm{s}$ and Tandem configuration the EDPCA SCNR improvement factor (IF) with respect to the single channel case (denoted by the blue circle markers) is around $3 \mathrm{~dB}$ and better when compared to the Boom configuration case. It can be theoretically demonstrated that for $v_{z}=0 \mathrm{~m} / \mathrm{s}$ and in the limit of zero clutter coherence between the different channels, IF tends to the number of channels $M$, since in this case clutter has no spatial structure and behaves like noise. As the worst clutter coherence is 0.15 according to (7) for the longest baseline and a $10 \mathrm{~ms}$ clutter coherence time, IF is expected to be lower than $\mathrm{M}$.

To complement the theoretical analysis, the EDPCA SCNR for different variations of the proposed Boom configuration is reported in Fig. 4 as a function of the across-track ground velocity. Apart from the considered Boom architecture (solid red line), three alternatives are analyzed: the first two (blue asterisk and green diamond solid lines) have the same configuration as the Boom system but with a mast length of $28.8 \mathrm{~m}$ and $57.6 \mathrm{~m}$, respectively; while the third system (black circles solid line), referred as $\mathrm{XDB}$, is a Boom configuration, where the two first channels are X-DRA like (2.4 m separation and no unfolding) and the third antenna is deployed with a mast of
TABLE II

Monte CARlo (MC) Simulation PaRAMETERs.

\begin{tabular}{lcc}
\hline Parameter & Value & Units \\
\hline RCS (step of $\left.1 \mathrm{dBm}^{2}\right)$ & -10 to 20 & $\mathrm{dBm}^{2}$ \\
$v_{z}($ step of $0.25 \mathrm{~m} / \mathrm{s})$ & 0 to 50 & $\mathrm{~m} / \mathrm{s}$ \\
Target type & Deterministic/Gaussian & \\
Sea state & 4 & \\
Clutter correlation & $5 \cdot 10$ & $\mathrm{~ms}$ \\
MC trials & $10^{-5}$ & \\
$P_{f a} a$ & \\
Window type & Kaiser (factor 2.5$)$ & \\
Azimuth/Range processing bandwidth & $2.8 \mathrm{e} 3 / 150 \mathrm{e} 6$ & $\mathrm{~Hz}$ \\
\hline
\end{tabular}

$14.4 \mathrm{~m}$. The proposed configuration (red solid line) provides an overall improved SCNR compared to the rest of systems. Increasing the mast separation allows a slightly narrower notch response (around zero velocity), at the expense of additional steeper secondary notches ("blind" velocities). Moreover, there is a progressive degradation in the SCNR peaks due to clutter decorrelation higher impact. For XDB a generally reduced SCNR is observed since the antenna dimensions are half of the Boom architecture. Secondary notches, much steeper, are located at slightly higher velocities because of effective shorter baselines.

\section{Simulation RESUlTS: PERFORMANCE EVALUATION}

\section{A. Monte Carlo approach}

The detection capabilities of any GMTI system can be statistically quantified through the probability of detection $P_{d}$. In this sense, MC simulations provide a very useful tool to asymptotically characterize this probability for specific system and scenario conditions. A flexible SAR-GMTI performance simulator tool, based on MC simulations at image level, has been implemented to provide statistical metrics in terms of $P_{d}$ and $P_{f a}$. This simulator avoids generating and processing iteratively synthetic raw data for a given system/scenario to characterize the $P_{d}$, which could otherwise be time-consuming.

In the MC simulations presented in this section some hypotheses have been assumed: (i) the clutter is a zero-mean complex Gaussian process with given spatial (along different channels) correlation properties; (ii) SAR processing has been adapted to the target kinematic parameters ${ }^{3}$; (iii) the residual phase error (due to coregistration mismatch) defined by the second term of (10) has been included for the first 440 combined ambiguities; (iv) theoretical covariance matrix in EDPCA processing is used; and (v) the target (either deterministic or Gaussian) is correlated from look to look, while clutter has been assumed uncorrelated. Table II summarizes the different parameters of the MC simulations considering the center of subswath 8 with an incidence angle of 33.17 degrees.

Fig. 5 shows probability of detection maps as a function of the RCS and ground range velocity for X-DRA system with ATI processing and Boom configuration with EDPCA technique, when considering a deterministic target and 10 ms clutter coherence time (no multilook processing). Boom

\footnotetext{
${ }^{3}$ Discussion about SAR focusing mismatch on fast moving boats is considered in section V-B.
} 


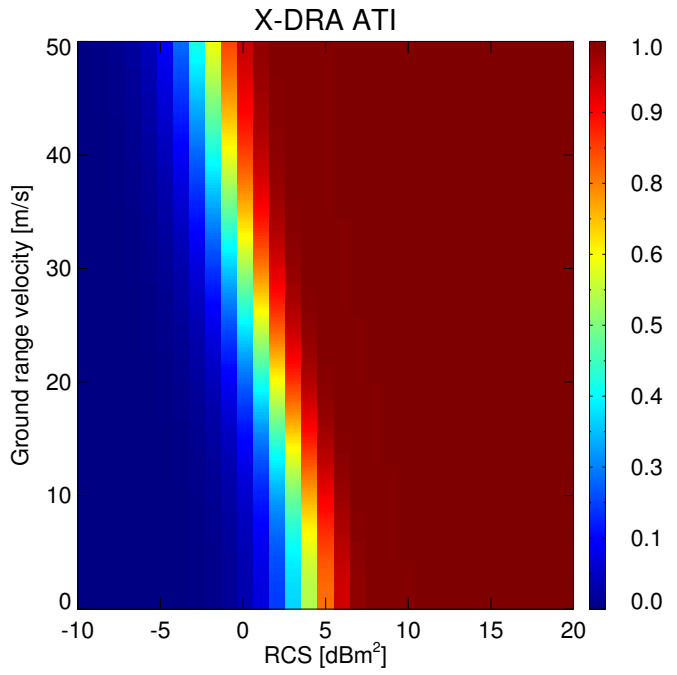

(a)

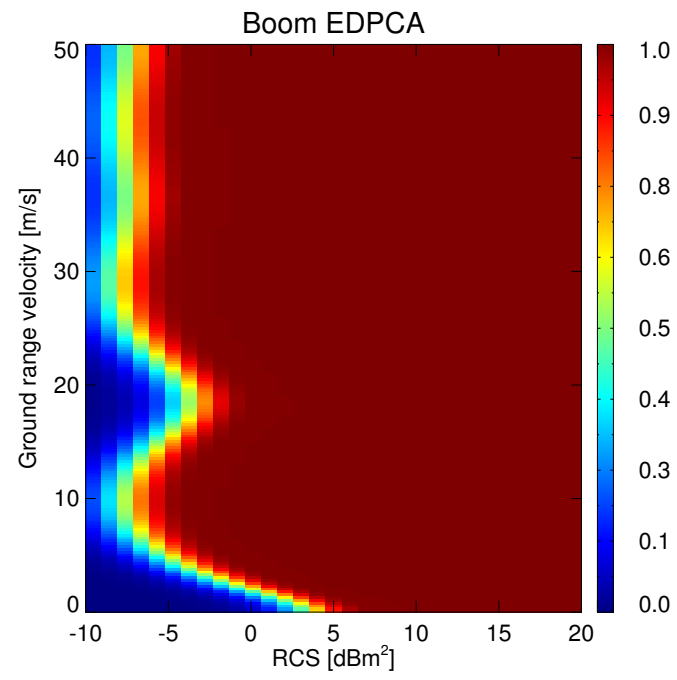

(b)

Fig. 5. Probability of detection maps (versus RCS and ground range velocity) obtained from MC simulations for different system-technique configurations: (a) ATI X-DRA and (b) EDPCA Boom (deterministic target, complex Gaussian clutter with 10 ms coherence time, no multilook and CFAR detectors with $P_{f a}$ of $10^{-5}$ ).

system in combination with EDPCA, Fig. 5(b), provides improved performance compared to the other system-technique combinations, especially in the region of slow and low reflectivity moving targets. DPCA technique applied to X-DRA data provides the worst $P_{d}$ in this region. However, the joint 2D phase-magnitude ATI detector on the X-DRA system, Fig. 5(a), gives comparatively a better performance, close to the EDPCA applied to Tandem system, where the induced higher clutter decorrelation is the driven parameter; but still EDPCA on Tandem has a threshold RCS, approximately $5 \mathrm{~dB}$ below the ATI case. In Fig. 5(b), the secondary notch around $20 \mathrm{~m} / \mathrm{s}$ for RCS is related to the degradation on SCNR, as observed from Fig. 3(b), caused by the presence of a blind velocity associated to the longer baseline.

Fig. 6(a) and Fig. 6(b) show cuts of the $P_{d}$ for the different system-technique combinations as a function of across-track ground velocity (for a RCS of $-5 \mathrm{dBm}^{2}$ ) and versus RCS (for $v_{z}=1 \mathrm{~m} / \mathrm{s}$ ), respectively. Two sea clutter coherence times ( $\infty$ and $10 \mathrm{~ms}$ ) are reported for each system-technique combination to illustrate the impact of clutter decorrelation. For a completely correlated sea clutter the Tandem configuration with EDPCA processing (solid blue line) provides the highest sensitivity to slowly moving targets, at the expense of a reduced unambiguous range of velocities. However, for realistic scenario operations clutter decorrelation impairs especially the performance of the Tandem system (solid blue line with asterisk); while Boom configuration (with $14.4 \mathrm{~m}$ mast) shows a robust behavior (comparing red dashed lines with and without asterisk markers), providing the best detection capabilities as a function of the across-track velocity for a small target. ATI (dotted black line) and DPCA (dash-dot-dot green line) for X-DRA prove to have very limited capabilities to detect boats with reduced reflectivity $\left(-5 \mathrm{dBm}^{2}\right)$, being almost insensitive to clutter decorrelation since X-DRA has the smallest baseline, $2.4 \mathrm{~m}$. When considering the performance as a function of the RCS for slow moving boats, DPCA technique for X-DRA provides the worst results, being unable to detect targets even with moderate to high reflectivity. For the same configuration, ATI 2D detector gives much better results, and gets closer to the Boom-EDPCA, which still requires lower RCS to obtain the same $P_{d}$. In this regard, and even for a 10 ms coherence time, Tandem configuration provides the lowest threshold RCS value, since the boat velocity is still in the range of $v_{z}= \pm 1.5 \mathrm{~m} / \mathrm{s}$, where as already observed in Fig. 3(b) the Tandem SCNR is around $3 \mathrm{~dB}$ higher.

Analogously, Fig. 7 shows the same $P_{d}$ cuts but for a worse case scenario, where the target has been modeled as complex Gaussian process (completely correlated from channel to channel). Comparing both situations, Fig. 6 and Fig. 7, target randomness provides in average a smaller $P_{d}$ as a function of $v_{z}$, smoothing the trend of $P_{d}$ as a function of the RCS. For the Gaussian target and for $P_{d}$ below a threshold level of 0.3 , improved performance is obtained w.r.t. deterministic case, whereas for values above this value there is a global degradation. Additionally, the impact of data averaging (multilook processing) on $P_{d}$ has been also evaluated (not reported here due to space limitations), showing an improvement on $P_{d}$ as the number of looks (boxcar size) increases, whenever the target occupies a sufficient number of single-look resolution cells to avoid degradation on the effective SCNR after multilook, see [13].

\section{B. SAR data approach}

To complement the MC simulations, synthetic multichannel data is generated using a flexible SAR-GMTI simulator tool, where different systems, modes of operation and scenarios can be easily configured [5], [7], [8].

In order to provide an analogous metric to the probability of detection, several raw data simulations (20 trials) have been carried out, such that a frequency of detection can be 


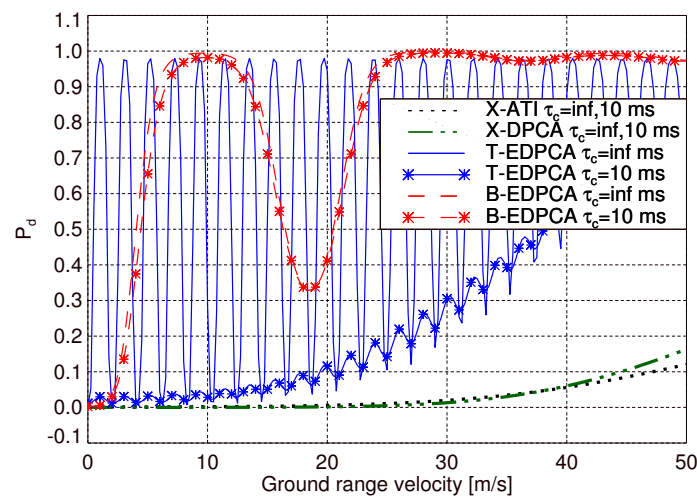

(a)

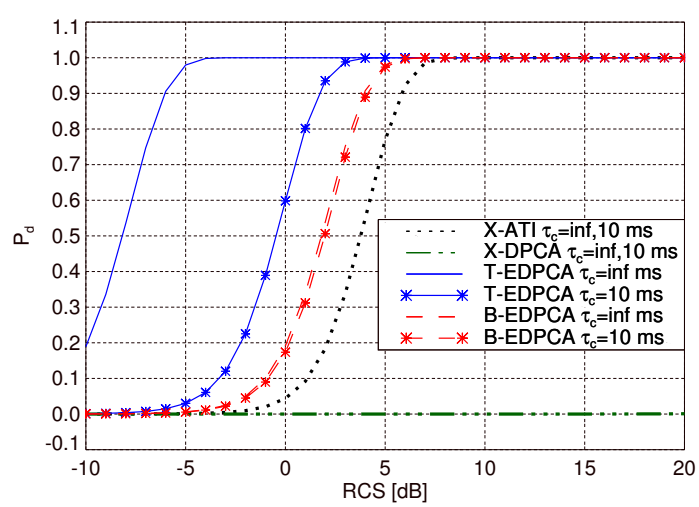

(b)

Fig. 6. System-technique comparison of the probability of detection obtained from MC simulations assuming a deterministic target and for different clutter correlations ( $\tau_{c}=$ inf and $10 \mathrm{~ms}$ ): (a) cut at RCS of $-5 \mathrm{dBm}^{2}$ as a function of ground velocity and (b) cut at $v_{z}=1 \mathrm{~m} / \mathrm{s}$ as a function of RCS; no multilook processing is considered (in legend $\mathrm{X}$ refers to X-DRA system, $\mathrm{T}$ and $\mathrm{B}$ to Tandem and Boom configurations, respectively).

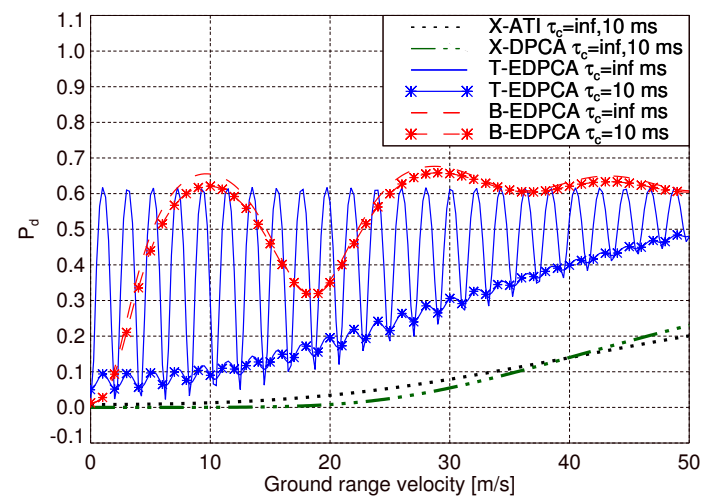

(a)

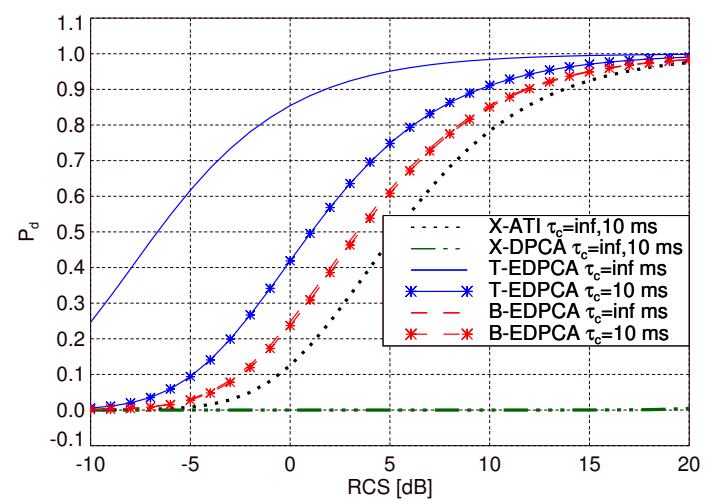

(b)

Fig. 7. System-technique comparison of the probability of detection obtained from MC simulations assuming a complex Gaussian target and for different clutter correlations $\left(\tau_{c}=\right.$ inf and $10 \mathrm{~ms}$ ): (a) cut at RCS of $-5 \mathrm{dBm}^{2}$ as a function of ground velocity and (b) cut at $v_{z}=1 \mathrm{~m} / \mathrm{s}$ as a function of RCS; no multilook processing is considered (in legend $\mathrm{X}$ refers to X-DRA system, $\mathrm{T}$ and $\mathrm{B}$ to Tandem and Boom configurations, respectively).

TABLE III

TARGET PARAMETERS

\begin{tabular}{lccccccccc}
\hline Target & Type & Scatterers mean RCS $\left[\mathrm{dBm}^{2}\right]$ max. RCS $\left[\mathrm{dBm}^{2}\right]$ & $v_{x}[\mathrm{~m} / \mathrm{s}]$ & $v_{z}[\mathrm{~m} / \mathrm{s}]$ & $a_{x}\left[\mathrm{~m} / \mathrm{s}^{2}\right]$ & $a_{y}\left[\mathrm{~m} / \mathrm{s}^{2}\right]$ & $a_{z}\left[\mathrm{~m} / \mathrm{s}^{2}\right]$ \\
\hline T1 & Ramshackle & 53 & 4.5 & 17 & -1.12 & 6.25 & -0.69 & 0.0 & -0.30 \\
T2 & Civil cargo & 102 & 28.2 & 40 & -6.73 & 3.0 & -0.22 & 0.0 & -0.29 \\
T3 & Point-like target & 1 & -5 & -5 & 0 & 5 & -0.69 & 0.0 & -0.4 \\
T4 & Point-like target & 1 & 0 & 0 & 0 & 2.5 & -0.69 & 0.0 & -0.4 \\
T5 & Point-like target & 1 & 5 & 5 & 20.6 & 0.0 & 2.45 & 3.43 & 0.0 \\
T6 & Point-like target & 1 & 5 & 5 & 0.0 & 20.6 & 0.0 & 3.43 & 2.45 \\
\hline
\end{tabular}

extracted. A complete processing chain has been implemented integrating a MFB (based on a RD processor) with the different GMTI techniques, similar to [19], [34]. Each filter performs an adaptive SAR processing, at both range cell migration correction (RCMC) and azimuth focusing steps, for a specific set of kinematic parameters $\left(v_{z}, a_{z}\right.$ and $\left.v_{x}\right)$, trying to recover a well focused moving object. No compensation of the Doppler shift (azimuth shift) is carried out, since, otherwise, the target will appear at different azimuth positions for different $v_{z}$ of the filter bank, posing difficulties to efficiently compare the outputs of the bank of filters. As in the MC simulations, a Kaiser window (factor 2.5) has been used in range and azimuth with processing bandwidths of $2.8 \mathrm{KHz}$ and $150 \mathrm{MHz}$, respectively.

A $1 \mathrm{Km}$ by $1 \mathrm{Km}$ scene extension (in ground) centered at $\gamma_{0}=33.17$ degrees has been simulated, considering a sea state $4(-15.1 \mathrm{~dB}$ normalized reflectivity) with $10 \mathrm{~ms}$ coherence time. In a first approach, a zero-mean complex Gaussian reflectivity for sea clutter has been assumed, in a downwind acquisition. To complement these results, and for the same sea conditions, a K-distributed clutter has been also considered, trying to emulate more realistic maritime scenarios. Six deterministic moving targets are included in the scenario as indicated in Table III, where two different type of vessels (Ramshackle T1 and a Civil cargo T2) have been modeled as a collection of point-like targets. The electromagnetic modeling and RCS extraction of these vessels have been carried out by Telespazio Vega UK company, in the cooperation frame of the European Commission FP7 funded Project SIMTISYS, [8]. In a first approximation, the targets 


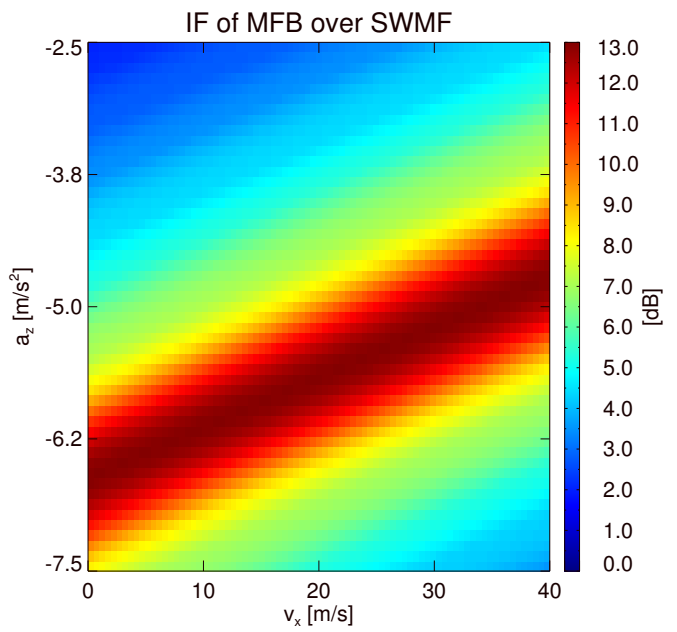

(a)

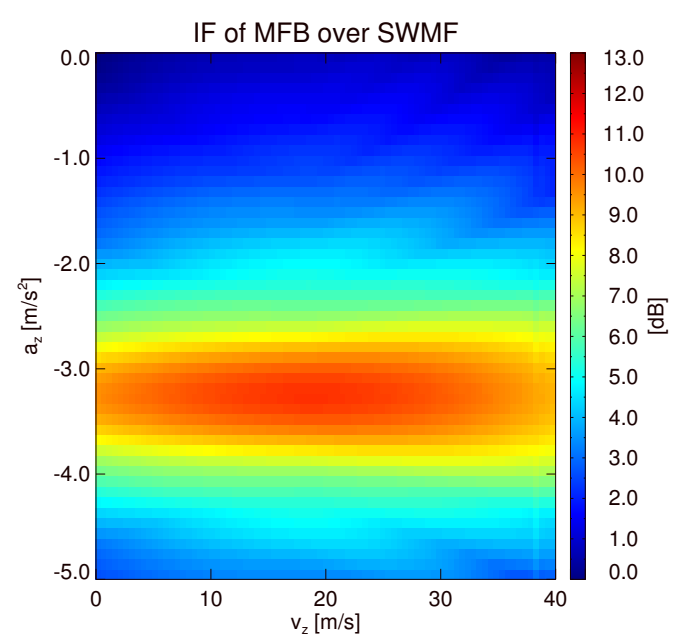

(b)

Fig. 8. Improvement factor of the MFB with respect to SWMF: (a) target T5 $v_{x}$ versus $a_{z}$ map; (b) target T6 $v_{z}$ versus $a_{z}$ map.

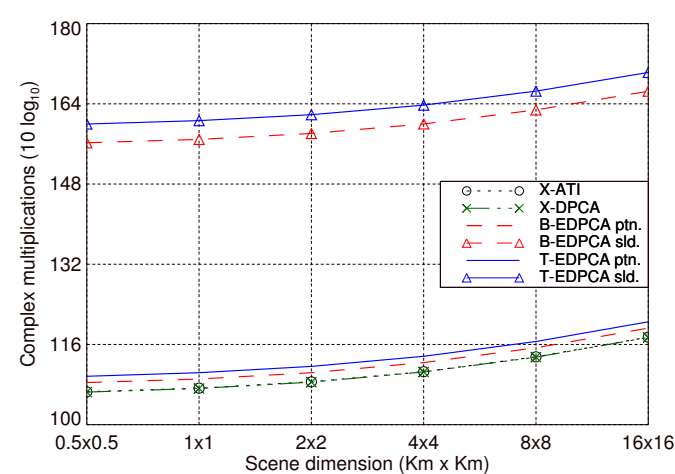

Fig. 9. Computational load comparison considering a MFB with 33 filters; in legend ptn. (portion) and sld. (sliding) refer to the type of approach followed to estimate/invert the interference covariance matrix in the EDPCA processing, using a single portion of the image (512x512 pixles) and a sliding boxcar (512x512 pixels size), respectively.

were simulated as rigid bodies. Targets $\mathrm{T} 3$ and $\mathrm{T} 4$ are single scattering points modeling low reflectivity slow moving targets in order to prove the potential improvement of the proposed Boom configuration. High-speed boats can reach velocities between 40-70 Knots (20.6-36 m/s) experiencing longitudinal and/or vertical accelerations up to several $g$-forces, depending on sea conditions, [35]. Point-targets T5 and T6 represent high-speed boats moving in along- and across-track directions, respectively, with vertical accelerations $a_{y}$ of $0.35 \mathrm{gm} / \mathrm{s}^{2}$ and longitudinal ones, $a_{x}=0.25 \mathrm{gm} / \mathrm{s}^{2}$ (T5) and $a_{z}=0.25 \mathrm{~g} \mathrm{~m} / \mathrm{s}^{2}$ (T6).

It is well-known that if target kinematic is not accounted for in the SAR processor, severe degradations are observed in the SAR image and this in turn can avoid proper SARGMTI detection, especially for small and fast moving objects. Fig. 8(a) and Fig. 8(b) show the IF maps provided by the MFB w.r.t. a stationary world matched filter (SWMF) for T5 and T6, respectively. In case of T5, with a purely along-track movement and no $a_{z}$ accelerations, the MFB can provide up to $13 \mathrm{~dB}$ of improvement for a set of $a_{z}-v_{x}$ pairs as denoted by the diagonal strip in Fig. 8(a). Two conclusions are extracted from these results: first, vertical acceleration $a_{y}$ on T5 produces equivalently similar effects as an $a_{z}$ acceleration from the SAR processing point of view since $a_{y}$ contributes also to a radial acceleration (line-of-sight projection). Second, there is a coupling between across-track acceleration $a_{z}$ and along-track velocity $v_{x}$ giving rise to indistinguishable effects. For a fixed $v_{x}$, a MFB step size below $0.5 \mathrm{~m} / \mathrm{s}^{2}$ on $a_{z}$, produces losses on peak response below $3 \mathrm{~dB}$. Analogously, for a fixed $a_{z}$ the step on $v_{x}$ is below $13 \mathrm{~m} / \mathrm{s}$. The impact of $a_{x}$, inducing third-order phase errors, produces mainly an asymmetric sidelobe response for the considered acquisition time (below one second).

For T6, moving in the across-track direction, the combination of $a_{z}$ and $a_{y}$ produces an effective across-track acceleration different from the target's original one (2.45 $\mathrm{m} / \mathrm{s}^{2}$ ), see Fig. $8(\mathrm{~b})$, with an IF around $11 \mathrm{~dB}$. Similar to T5, a MFB step size below $0.5 \mathrm{~m} / \mathrm{s}^{2}$ on $a_{z}$ produces losses below $3 \mathrm{~dB}$, while the sensitivity w.r.t. $v_{z}$ is quite flat, with a degradation around $2 \mathrm{~dB}$ for a variation of $20 \mathrm{~m} / \mathrm{s}$. It must be noted that azimuth processing is performed around the target's Doppler centroid with the given processed bandwidth, and so if there is any backfolding of target's spectrum (Doppler ambiguous velocity), the processing window should be properly adapted to maximize the spectral overlap, reducing the defocusing effects. The adaptive SAR processor has been derived assuming uniformly accelerated movements for the vessels during the synthetic aperture time, considering second order Taylor's series expansion for the slant range history. However, such an assumption is too strict especially for small and high-speed boats. Therefore, in realistic scenarios, the proposed adaptive SAR processor would not be able to fully recover the induced image degradations, but this is out of the paper's scope. Alternative SAR processing strategies should be considered as well as realistic modeling of the complex dynamics of high-speed boats on open seas.

The required computational cost of the implemented processing chain is represented in Fig. 9 in terms of complex multiplications as a function of the scene dimension. In 


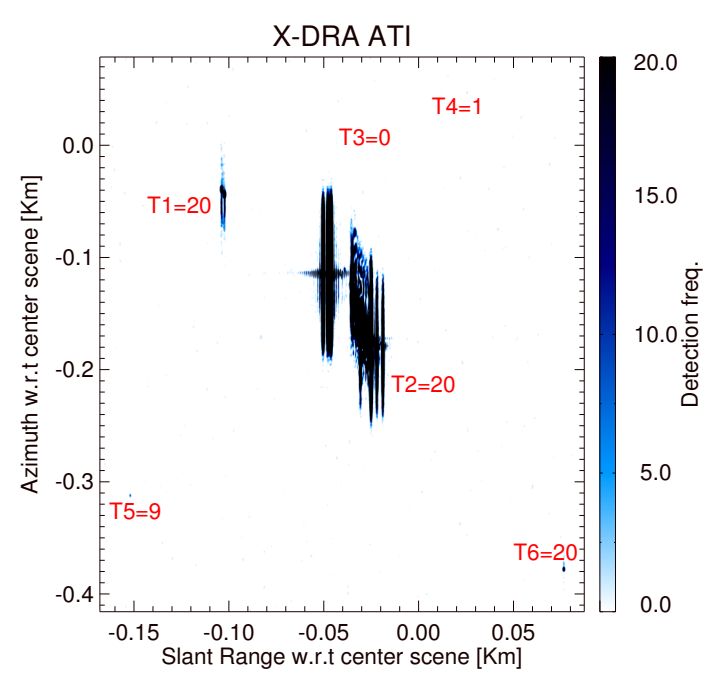

(a)

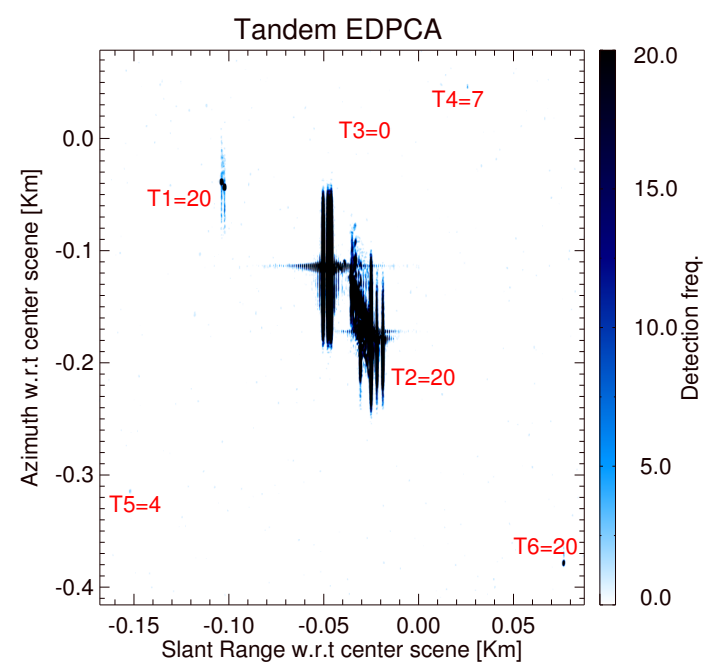

(b)

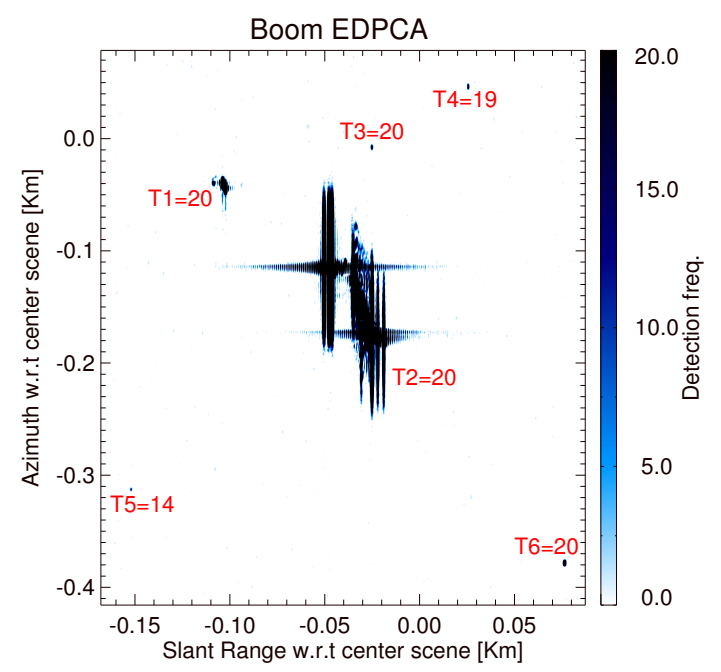

(c)

Fig. 10. Combined detection maps assuming a complex Gaussian distributed sea clutter (number of detections per target detailed): (a) XDRA architecture using ATI; (b) Tandem and (c) Boom configurations with EDPCA (CFAR detectors with $P_{f a}=10^{-5}$ under Gaussian hypothesis).

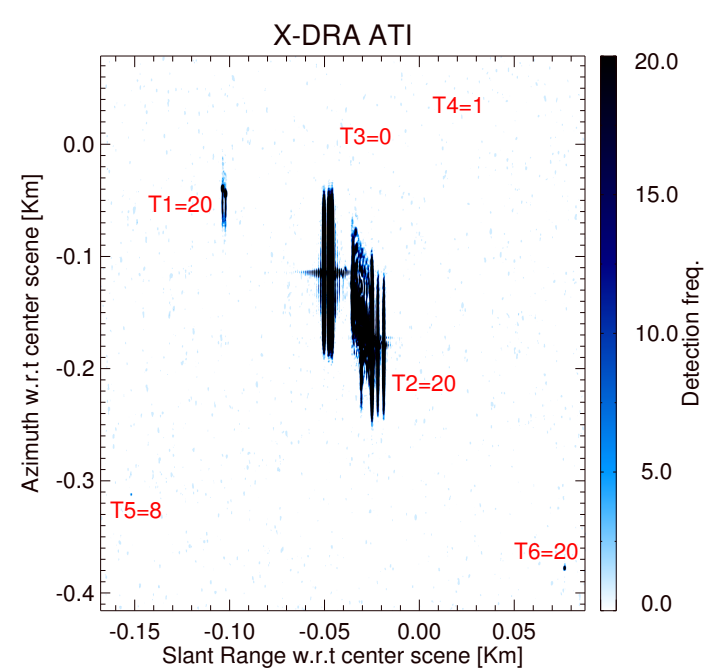

(a)

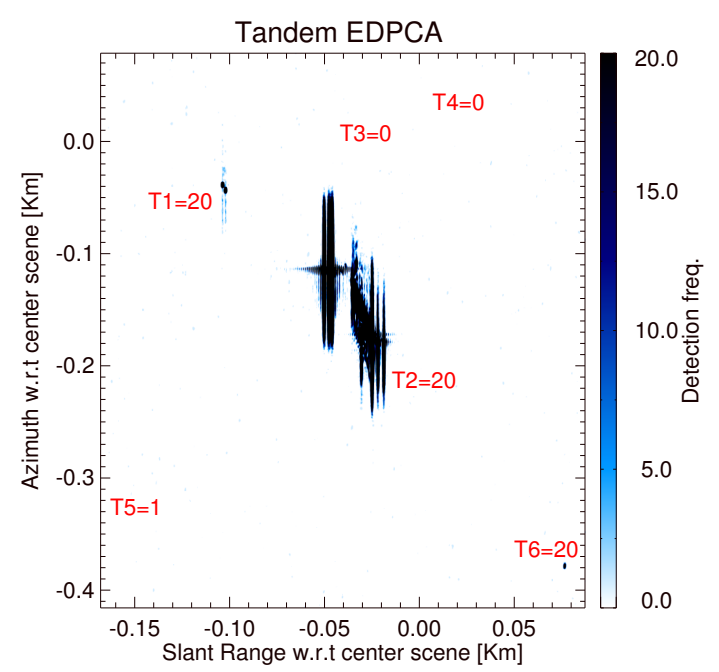

(b)

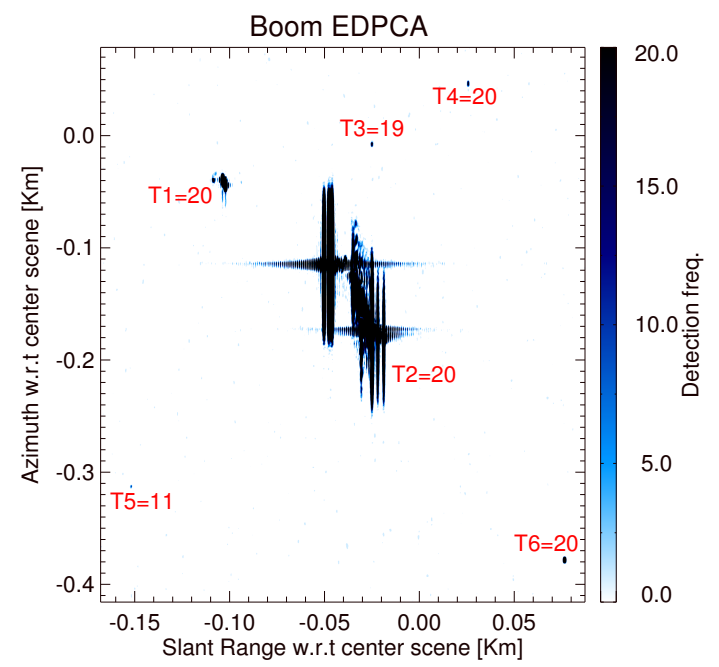

(c)

Fig. 11. Combined detection maps assuming a K-distributed sea clutter (number of detections per target detailed): (a) X-DRA architecture using ATI (2D-CFAR under Gaussian hypothesis with $P_{f a}=10^{-5}$ ); (b) Tandem and (c) Boom configurations with EDPCA (1D-CFAR under K-distribution hypothesis with $P_{f a}=10^{-5}$ ). 
this case, 33 filters have been used to sample the range of $a_{z}= \pm 10.0 \mathrm{~m} / \mathrm{s}^{2}$ with a step of $0.5 \mathrm{~m} / \mathrm{s}^{2}$, producing a degradation below $3 \mathrm{~dB}$ on the peak response. For X-DRA configuration ATI and DPCA techniques (lines with circle and cross markers) have the lowest computation burden compared to EDPCA, where the required number of operations increases with the number of additional channels and especially when considering a sliding boxcar (pixel by pixel basis) approach for $\mathbf{R}_{q}$ estimation/inversion.

The combined detection maps over the 20 trials are represented in Fig. 10 and Fig. 11 assuming a complex Gaussian and a K-distributed (shape parameter of $v=5$ ) sea clutter, respectively. The combined detection map per trial is obtained as the aggregation of the different detection images (each adapted to each one of the six targets) through the logical operator $O R$. It must be noted that the strips (along azimuth) of detected pixels around $\mathrm{T} 1$ and especially $\mathrm{T} 2$ are present because of the defocusing induced by the matched filters adapted to T5 and T6, such that those pixels are still above the threshold established by the CFAR detector.

From Fig. 10 and Fig. 11 it can be generally stated that T1, $\mathrm{T} 2$ and $\mathrm{T} 6$ targets are well detected by the different systemtechnique combinations (also for X-DRA with DPCA) over the various trials. Considering X-DRA configuration, small and slowly moving targets $\mathrm{T} 3$ and $\mathrm{T} 4$ are neither detected by the ATI, see Fig. 10(a), nor by DPCA (not reported here due to space limitations), for which $\mathrm{MC} P_{d} \mathrm{~s}$ are close to zero. Unlike DPCA, ATI is able to provide around 9 detections for T5 (MC estimates a $P_{d}$ of 0.76 ). When considering a K-distributed clutter, Fig. 11(a), the overall detection performance is maintained at the expense of increased false alarms (see Table IV), since the parametric 2D-CFAR detector still assumes a Rayleigh distributed magnitude on the clutter; however, as there is no clutter cancellation such hypothesis breaks down. Further studies are required to theoretically derive a 2D-ATI parametric detector considering a K-distributed clutter in magnitude (or more generally a compound model statistical family).

In EDPCA-Tandem, Fig. 10(b), T3 is not at all detected and T4 is gathered in 7 out of 20 trials, under the assumption of a complex Gaussian clutter, as predicted by $\mathrm{MC}$ with $P_{d} \mathrm{~s}$ of 0.03 and 0.43 , respectively. For a K-distributed sea clutter, Fig. 11(b), neither T3 nor T4 are detected using EDPCATandem combination with a CFAR detector fitted to a Kdistribution. For this case the required threshold (to keep the same $P_{f a}$ ) is higher compared to Rayleigh case.

The low reflectivity slow point-like targets $\mathrm{T} 3$ and $\mathrm{T} 4$ are well detected (almost in all trials) applying EDPCA with the proposed Boom configuration, Fig. 10(c), according to the predicted $P_{d}$ (0.65 and 0.82$)$ from MC simulations. The number of detections over the reduced set of trials for the raw data processing approach provide a general picture on the expected performance, but cannot be directly compared to the intensive MC simulation $P_{d} \mathrm{~s}$, which have been computed using 5 millions of iterations. EDPCA with Boom configuration appears to be more robust to the clutter statistical variation, comparing Fig. 10(c) and Fig. 11(c), since the overall detection performance is kept, specifically for T3 and T4.

MC simulations predict a $P_{d}$ around 1 for target T5 and
TABLE IV

ESTIMATED $P_{f a}$ FOR DIFFERENT TECHNIQUES, CLUTTER AND CFAR STATISTICS (AVERAGED OVER 20 TRIALS AND 6 FILTERS).

\begin{tabular}{cccc}
\hline & \multirow{2}{*}{ Gaussian } & \multicolumn{2}{c}{ K-distributed } \\
\cline { 3 - 4 } & & Rayleigh CFAR & K-distributed CFAR \\
\hline X-DRA DPCA & $1.05 \mathrm{e}-5$ & $1.05 \mathrm{e}-5$ & $1.05 \mathrm{e}-5$ \\
X-DRA ATI & $1.06 \mathrm{e}-5$ & $1.70 \mathrm{e}-4$ & - \\
Tandem EDPCA & $1.14 \mathrm{e}-5$ & $2.0 \mathrm{e}-4$ & $1.40 \mathrm{e}-5$ \\
Boom EDPCA & $1.14 \mathrm{e}-5$ & $4.44 \mathrm{e}-5$ & $1.88 \mathrm{e}-5$ \\
\hline
\end{tabular}

Tandem configuration using EDPCA processing. However, from Fig. 10(b), the number of detections is 4, appearing at a displaced azimuth position compared to the other systemtechnique combinations. From the inspection of the individual coregistrated SAR images, an azimuth displacement on the T5 peak response can be recognized, around $4 \mathrm{~m}$ for the furthest apart channels. This shift is due mainly to the high vertical acceleration $a_{y}$ and partially to the along-track velocity $v_{x}$. In fact, $a_{y}$ translates into a radial acceleration, which produces a variation on the effective radial velocity (during the coregistration time or equivalently the baseline time delay) observed by the different channels, producing a different azimuth displacement between channels. Such statement has been confirmed by additional simulations, where the same T5 kinematics with no $a_{y}$ have been considered, and a slight shift is observed between target peak responses due to $v_{x}$. Therefore, the azimuth displacement (mostly caused by $a_{y}$ ) avoids the full coherent integration of EDPCA, since target response appears on different pixels for different channels, reducing the detection capabilities compared to MC simulations, where it has been ideally assumed that target response among receivers lies on the same resolution cell. For the more compact Boom architecture, such a displacement is below $1.0 \mathrm{~m}$, keeping T5 peak responses within the same resolution cell, in a way that EDPCA processing is still effective providing 14 detections out of 20, in line with the predicted $P_{d}$ of 0.84 .

Table IV presents the average (over the 20 trials and 6 filters) estimated $P_{f a}$ for the considered system-technique combinations and for the two types of clutter statistics, complex Gaussian (2nd column) and K-distributed in magnitude (3rd and 4th columns). For the latter case two CFAR parametrization are evaluated, Rayleigh (3rd column) and K-distribution (4th column), only for DPCA and EDPCA. As expected, DPCA processing on X-DRA keeps the same level of false alarms for the different clutter statistics and CFAR settings. However, for ATI processing, $P_{f a}$ is one order of magnitude higher for the K-distributed clutter when a 2D-CFAR detector under the assumption of Gaussian-like interference is used. Similarly, EDPCA processing on Tandem and Boom present a higher $P_{f a}$ (especially Tandem due to clutter decorrelation) when using a Rayleigh CFAR detector for the K-distributed scenario; but it gets reduced for the most appropriate Kdistributed CFAR scheme.

\section{Conclusions}

The GMTI performance of different multichannel SAR missions using state-of-the-art GMTI techniques (ATI, DPCA and EDPCA) has been evaluated in this paper over maritime 
scenarios. A new multichannel SAR configuration, based on non-uniformly displaced receive phase centers on-board a single satellite with deployable antennas, has been compared with TSX and TDX like missions. With such multichannel configuration it is possible to discern small slow moving vessels, which are usually undetected with present SAR systems. Under certain hypothesis (homogeneous open sea), the proposed mission tries to maximize the SAR-GMTI performance by minimizing the noise contribution, in terms of NESZ, and ambiguities' level in the region of interest (2040 degrees of incidence angle), while keeping a low system complexity with an optimized number of channels. Boom system has been designed trying to keep the new ambiguity metric CRAASR as low as possible in the region of operation. In the far range region (40-60 degrees) the impact of range ambiguities increases and so does CRAASR. Therefore, the performance is expected to degrade for the case of nonhomegeneous ambiguous returns, especially close to coastal areas. A way to circumvent these impairments is to consider a future SAR-GMTI mission concept exploiting azimuth phase coding (APC) in combination with digital beamforming, such that a high-resolution wide swath (HRWS) mode can be operated [36].

The proposed architecture has been evaluated comparatively in a two-level simulation performance approach over maritime scenarios. In a first step, the probability of detection has been characterized through intensive MC simulations under different scenario conditions. Several synthetic multichannel SAR raw data sets have been processed iteratively to complement MC simulations for a realistic maritime scenario. The different simulations' results indicate the potential improvement provided by the proposed Boom configuration when using adaptive processing approaches such as EDPCA. The simulations results are in good agreement with the theoretical expected performance. Boom configuration has been designed, such that it is slightly affected by internal clutter motion and at the same time enables high sensitivity to low reflectivity slow moving targets, alleviating Doppler (velocity) ambiguities thanks to its baseline diversity. It must be pointed out that this configuration can be scaled down (in terms of size) for a higher operating frequency, with the appropriate modification of the sea clutter modeling (in terms of normalized reflectivity and correlation).

The performance evaluation of the proposed mission has been carried out assuming a complex Gaussian model for the sea clutter. Additional raw data simulations assuming a K-distributed sea clutter, have been performed, showing the robustness of Boom configuration when processed with EDPCA. For ATI, where no clutter cancellation is performed, a new formulation of the 2D-CFAR detector should be derived to consider a K-distributed sea clutter. Additionally, the impact of SAR imaging high-speed boats has been preliminary analyzed, showing the need to properly account for target kinematics in the SAR-GMTI processing chain via a MFB since, otherwise, the induced image degradation could impair the GMTI performance, particularly for small and fast boats. From these considerations, it can be stated that experimental campaigns over maritime scenarios are mandatory to understand and validate a sea clutter model (for the usual range of SAR incidence angles), in terms of its statistics and mean power reflectivity, and at the same time try to grasp the impact of realistic target kinematics.

\section{ACKNOWLEDGMENT}

The authors would like to thank Alasdhair Beaton from Telespazio Vega UK for providing the vessel models. The authors would also like to thank Dr. Santiago Capdevila and Aglaia Gómez for their helpful suggestions and comments that have strongly contributed to the clarity of this paper.

\section{REFERENCES}

[1] R. Werninghaus and S. Buckreuss, "The TerraSAR-X Mission and System Design," IEEE Trans. Geosci. Remote Sens., vol. 48, no. 2, pp. 606-614, 2010.

[2] G. Krieger, A. Moreira, H. Fiedler, I. Hajnsek, M. Werner, M. Younis, and M. Zink, "TanDEM-X: A Satellite Formation for High-Resolution SAR Interferometry," IEEE Trans. Geosci. Remote Sens., vol. 45, no. 11, pp. 3317-3341, 2007.

[3] M. Di Lazzaro, G. Angino, M. Piemontese, A. Capuzi, and R. Leonardi, "COSMO-SkyMed: The Dual-Use Component of a Geospatial System for Environment and Security," in IEEE Aerospace Conference, 2008, 2008, pp. $1-10$.

[4] A. Hillman, P. Rolland, R. Periard, A. Luscombe, M. Chabot, C. Chen, and N. Martens, "RADARSAT-2 initial system operations and performance," in Proc. IEEE IGARSS, vol. 2, 2009, pp. II-753 -II-756.

[5] E. Makhoul, A. Broquetas, and O. Gonzalez, "Evaluation of state-ofthe-art GMTI techniques for future spaceborne SAR systems -simulation validation," in Proc. EUSAR, April 2012, pp. 376-379.

[6] D. Cerutti-Maori, I. Sikaneta, and C. Gierull, "Ship detection with spaceborne multi-channel SAR/GMTI radars," Proc. EUSAR, pp. 400 -403 , april 2012.

[7] E. Makhoul, A. Broquetas, and J. Ruiz Rodon, "Ground moving target indication using multi-channel SAR with non-uniform displaced phase centers," in Proc. IEEE IGARSS, 2012, pp. 1610-1613.

[8] E. Makhoul, Y. Zhan, F. Ceba, A. Broquetas, A. Beaton, F. Letterio, S. Tonetti, S. Barbarossa, P. Di Lorenzo, and M. Maffei, "Fast Simulation Performance Evaluation of Spaceborne SAR-GMTI Missions for Maritime Applications," in Proc. EUSAR, June 2014.

[9] J. Ender, "Space-time processing for multichannel synthetic aperture radar," Elect. Commun. Eng. J., vol. 11, no. 1, pp. 29 -38, Feb 1999.

[10] C. Gierull, "Statistical analysis of multilook SAR interferograms for CFAR detection of ground moving targets," IEEE Trans. Geosci. Remote Sens., vol. 42, no. 4, pp. 691 - 701, April 2004.

[11] S. Baumgartner and G. Krieger, "Acceleration-independent along-track velocity estimation of moving targets," IET Radar, Sonar Navigation, vol. 4, no. 3, pp. $474-487$, jun. 2010.

[12] S. Chiu and C. Livingstone, "A Comparison of Displaced Phase Centre Antenna and Along-Track Interferometry Techniques for RADARSAT-2 Ground Moving Target Indication," in Can. J. Remote Sens., May 2005, vol. 31 , no. 1 , pp. 37-51.

[13] C. Gierull, I. Sikaneta, and D. Cerutti-Maori, "Two-Step Detector for RADARSAT-2's Experimental GMTI Mode," IEEE Trans. Geosci. Remote Sens., vol. 51, no. 1, pp. 436-454, 2013.

[14] J. Ender, C. Gierull, and D. Cerutti-Maori, "Improved Space-Based Moving Target Indication via Alternate Transmission and Receiver Switching," IEEE Trans. Geosci. Remote Sens., vol. 46, no. 12, pp. $3960-3974$, dec. 2008.

[15] D. Cerutti-Maori and J. Ender, "Performance analysis of multistatic configurations for spaceborne GMTI based on the auxiliary beam approach," IEE Proc. Radar, Sonar and Navig., vol. 153, no. 2, pp. 96 -103, April 2006.

[16] C. H. Gierull, D. Cerutti-Maori, and J. Ender, "Ground Moving Target Indication With Tandem Satellite Constellations," IEEE Geosci. Remote Sens. Lett., vol. 5, pp. 710-714, Oct. 2008.

[17] M. Gabele, S. Baumgartner, G. Krieger, and K.-H. Bethke, "Comparison of System Concepts for Traffic Monitoring with Multichannel SAR," Proc. EUSAR, 2006. 
[18] D. Cerutti-Maori, I. Sikaneta, and C. Gierull, "Optimum SAR/GMTI Processing and Its Application to the Radar Satellite RADARSAT-2 for Traffic Monitoring,” IEEE Trans. Geosci. Remote Sens., vol. 50, no. 10, pp. $3868-3881$, oct. 2012.

[19] D. Cerutti-Maori and I. Sikaneta, "A Generalization of DPCA Processing for Multichannel SAR/GMTI radars," IEEE Trans. Geosci. Remote Sens., vol. 51, no. 1, pp. 560-572, 2013.

[20] K. D. Ward, R. J. Tough, and S. Watts, Sea Clutter: Scattering, the K Distribution and Radar Performance. The Institution of Engineering and Technology, 2006.

[21] E. Makhoul, Y. Zhan, A. Broquetas, J. Ruiz Rodon, and S. Baumgartner, "Sea Clutter Statistical Characterization Using TerraSAR-X Data," in Proc. IEEE IGARSS, 2014.

[22] I. Cumming and F. Wong, Digital Processing Of Synthetic Aperture Radar Data: Algorithms And Implementation, ser. The Artech House Remote Sensing Library. Artech House, 2005.

[23] J. Sharma, C. Gierull, and M. Collins, "The influence of target acceleration on velocity estimation in dual-channel SAR-GMTI," IEEE Trans. Geosci. Remote Sens., vol. 44, no. 1, pp. 134 - 147, jan. 2006.

[24] C. H. Gierull, "Digital Channel Balancing of Along-Track Interferometric SAR Data," Defence R\&D Canada - Ottawa, Ottawa ONT (CAN), Tech. Rep., 2003.

[25] K.-H. Bethke, S. Baumgartner, M. Gabele, D. Hounam, E. Kemptner, D. Klement, G. Krieger, and R. Erxleben, "Air- and spaceborne monitoring of road traffic using SAR moving target indication-Project TRAMRAD," ISPRS J. Photogramm. Remote Sens., vol. 61, no. 3-4, pp. 243 - 259, 2006, theme Issue: Airborne and Spaceborne Traffic Monitoring.

[26] A. Budillon, V. Pascazio, and G. Schirinzi, "Estimation of Radial Velocity of Moving Targets by Along-Track Interferometric SAR Systems," IEEE Geosci. Remote Sens. Lett., vol. 5, no. 3, pp. 349 -353, july 2008.

[27] F. Lombardini, F. Bordoni, and F. Gini, "Feasibility study of alongtrack SAR interferometry with the COSMO-Skymed satellite system," in Proc. IGARSS, vol. 5, 2004, pp. 3337-3340 vol.5.

[28] V. Gregers-Hansen and R. Mital, "An improved empirical model for radar sea clutter reflectivity," IEEE Trans. Aerosp. Electron. Syst., vol. 48, no. 4, pp. 3512-3524, 2012.

[29] S. Frasier and A. Camps, "Dual-beam interferometry for ocean surface current vector mapping," IEEE Trans. Geosci. Remote Sens., vol. 39, no. 2, pp. 401-414, 2001

[30] S. Chiu, "A constant false alarm rate (CFAR) detector for RADARSAT-2 along-track interferometry," in Can. J. Remote Sens., May 2005, vol. 31, no. 1 , pp. 73-84.

[31] A. Farina and P. Lombardo, "Space-time techniques for SAR," in Applications of Space-Time Adaptive Processing, ser. IEE Radar, Sonar and Navigation, R. Klemm, Ed. The Institution of Electrical Engineers, 2004.

[32] R. Klemm, Space-Time Adaptive Processing: Principles and Applications. Institution of Engineering and Technology, 2002.

[33] J. C. Curlander and R. N. McDonough, Synthetic Aperture Radar: Systems and Signal Processing. Wiley, John \& Sons Incorporated, 1991.

[34] D. Cristallini, D. Pastina, F. Colone, and P. Lombardo, "Efficient Detection and Imaging of Moving Targets in SAR Images Based on Chirp Scaling," IEEE Trans. Geosci. Remote Sens., vol. 51, no. 4, pp. 2403-2416, 2013

[35] D. Savitsky and P. W. Brown, "Procedures for hydrodynamic evaluation of planing hulls in smooth and rough water," Marine Technology, vol. 13, no. 4, pp. 381-400, 1976.

[36] F. Bordoni, M. Younis, and G. Krieger, "Ambiguity Suppression by Azimuth Phase Coding in Multichannel SAR Systems," IEEE Trans. Geosci. Remote Sens., vol. 50, no. 2, pp. 617-629, 2012.

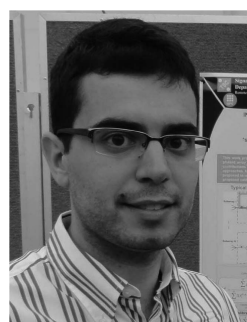

Eduard Makhoul (S'09) was born in Homs, Siria, in 1985. He received the Ingeniero degree in telecommunication engineering and the Master degree from the Universitat Politècnica de Catalunya (UPC), Barcelona, Spain, in 2009.

In 2008 he worked on his master thesis in the frame of HRWS-SAR systems at the German Aerospace Center (DLR). In 2009, he joined the Remote Sensing Laboratory, UPC, where is currently a Ph.D. candidate, researching on aspects of GMTI for future spaceborne SAR systems. From 2009 to 2011 he worked on the radiometric budget analysis of the Spanish mission PAZ. Since 2010 he is working in the analysis and study of SAR-GMTI systems and techniques for future European missions in the frame of NEWA and SIMTISYS projects. In 2013 (April-August) he was a visiting researcher at the DLR, working on SAR-GMTI processing of airborne and spaceborne data over maritime scenarios. His main research interests include SAR, GMTI, radar system design, calibration and array signal processing.

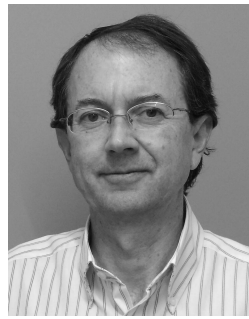

Antoni Broquetas (S'84, $\mathrm{M}^{\prime} 90$ ) was born in Barcelona, Spain, in 1959. He received the Ingeniero degree in telecommunication engineering from UPC in 1985, and the Doctor Ingeniero degree in 1989 in telecommunications engineering for his work on microwave tomography in the UPC.

In 1986 he was a Research Assistant in the Portsmouth Polytechnic (U.K.) involved in propagation studies. In 1987 he joined the Department of Signal Theory and Communications (TSC) of the UPC in Barcelona. From 1998 to 2002 he was Subdirector of Research at the Institute of Geomatics (Barcelona). From 1999 he is Full Professor at the UPC involved in research on radar imaging and remote sensing. From 2003 to 2006 he was Director of the TSC at UPC. He has published more than 150 papers on Microwave Tomography, Radar, ISAR and SAR systems, SAR processing and Interferometry.

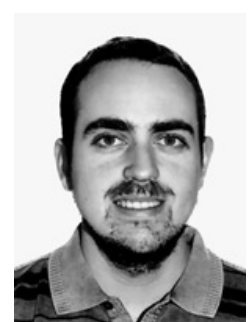

Josep Ruiz Rodon was born in Tarragona, Spain, in 1986. He received the degree in Telecommunication engineering from the UPC, Spain, in 2009; and the Master degree from Signal Theory and Communications Department in 2011. He is currently working toward the $\mathrm{Ph}$. D. degree in the TSC department at the UPC.

$\mathrm{He}$ has been working on SAR since January 2009, studying advanced scanning techniques for these systems, in particular TOPSAR mode, and its feasibility to the mission PAZ. He is involved in analysis of future SAR mission such as Geosynchronous SAR (GEOSAR) and Atmospheric Phase Screen (APS) retrieval studies.

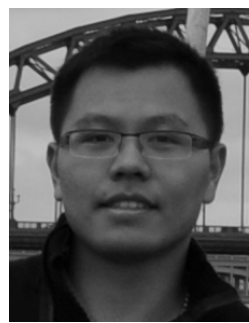

Yu Zhan was born in Zhejiang, China, in 1987. He received the degree in Telecommunication engineering from the UPC, Spain, in 2012, and the Master Degree from TSC department in 2013

In 2012, he joined the Remote Sensing Laboratory, at the UPC, where is currently a research assistant, involved on sea backscatter modeling for SAR systems, under the framework of SIMTISYS project. From 2012 to early 2013 he worked on the C-band polarimetric snow scattering analysis on mountainous areas, funded by the Spanish CDTI, project EOSWAN. His main research interests include SAR and multidimensional SAR, radar polarimetry, digital signal processing, GMTI and sea modeling.
Francisco Ceba was born in Barcelona, Spain, in 1988. He received the degree in Telecommunication engineering after a three-years degree from the UPC, Spain, in 2009. In September 2012 he joined the Remote Sensing Laboratory, at the UPC, as a research assistant, where is currently working on his thesis about GMTI under the framework of SIMTISYS project. 